\section{Does CPAP delay the development of cardiovascular disease in patients with obstructive sleep apnoea hypopnoea?} N T Ayas, G B J Mancini, J Fleetham

\section{Large, long term, randomised clinical trials are needed to establish whether CPAP treatment of patients with OSAH has a cardiovascular benefit}

$\mathrm{O}$ bstructive sleep apnoea hypopnoea (OSAH) is a common under-recognised disorder characterised by recurrent upper airway collapse during sleep. ${ }^{1}$ These recurrent episodes of upper airway collapse lead to sleep fragmentation, oxyhaemoglobin desaturation, and excessive daytime sleepiness. OSAH also causes sustained activation of the sympathetic nervous system, systemic inflammation with increased levels of C-reactive protein (CRP) and interleukin $6,{ }^{2}$ glucose intolerance, and endothelial dysfunction. ${ }^{3}$ Many of these physiological/biochemical abnormalities are implicated in the pathogenesis of cardiovascular disease (CVD). Indeed, there are compelling epidemiologic data implicating OSAH in the development of systemic hypertension, myocardial infarction, and cerebrovascular events.

OSAH is an independent risk factor for CVD. In a recent study from the Sleep Heart Health Cohort the prevalence of CVD (including myocardial infarction, angina, coronary revascularization, heart failure, stroke) was 1.42 times greater in patients with $\mathrm{OSAH}$ (apnoea hypopnoea index (AHI) $>11$ events/hour) than in those without OSAH (AHI 0-1.3 events/hour) after controlling for potential confounders. ${ }^{4}$ Furthermore, in a community based study, patients with moderate to severe OSAH were 2.89 times more likely to develop systemic hypertension over a 5 year period than patients without OSAH. ${ }^{5}$ Similarly, Mooe et al ${ }^{6}$ followed 408 subjects with coronary disease diagnosed by cardiac catheterisation. Patients with OSAH had a much higher risk of the composite outcome (death, stroke, transient ischaemic attack, myocardial infarction) than patients without OSAH $(26.3 \% v 16.2 \%)$ when followed for an average of 5.1 years.

Continuous positive airway pressure (CPAP) is the primary treatment for $\mathrm{OSAH}^{7}{ }^{7}$ It is very effective in eliminating upper airway collapse during sleep, improving sleep fragmentation, and decreasing daytime sleepiness. ${ }^{8}$ There are accumulating data that CPAP may reduce risks of CVD in patients with OSAH. CPAP treatment has beneficial effects on a variety of biochemical/ physiological markers known to be risk factors for CVD. Patients with OSAH have an increased prevalence of systemic hypertension and treatment with CPAP results in modest reductions in blood pressure. Pepperell and co-workers randomised 118 patients with OSAH to CPAP or subtherapeutic CPAP. ${ }^{9}$ Treatment with CPAP for 1 month reduced mean arterial 24 hour blood pressure by $2.5 \mathrm{~mm} \mathrm{Hg}$ and similar results have been found by other investigators. ${ }^{10}{ }^{11}$ If these findings persist long term, then CPAP may reduce future risks of CVD.

Systemic inflammation has recently been shown to be important in the pathogenesis of CVD. A number of studies have shown that CRP is an independent predictor of cardiovascular death, and CRP levels are now used to stratify the intensity of lipid lowering treatment. ${ }^{12}$ CRP levels are reduced by statins, drugs which have been shown to reduce the risks of CVD outcomes in large randomised controlled trials. ${ }^{13} \mathrm{CRP}$ levels are increased in patients with OSAH. Yokoe et al $^{14}$ reported that patients with OSAH had higher CRP levels than obese controls. Furthermore, CRP was correlated with AHI after controlling for body mass index. CRP levels decreased from 0.29 to $0.11 \mathrm{mg} / \mathrm{dl}$ in 17 patients with severe OSAH after 1 month of CPAP treatment. Although these data suggest that CPAP reduces CRP levels in patients with severe OSAH, the small numbers, short study period, and lack of a control group make it difficult to make definitive conclusions. To add to this controversy, a recent study of 47 patients with OSAH found no significant effect of CPAP on CRP levels. ${ }^{15}$ Preliminary data suggest that CPAP may improve lipid profiles and insulin sensitivity, both of which are risk factors for the development of CVD. Harsch and colleagues recently reported improvements in insulin sensitivity assessed by euglycaemic clamp methodology in 31 patients with OSAH treated with CPAP for 3 months. ${ }^{16}$ Robinson and Co-workers also found a significant fall in serum cholesterol levels after 1 month of treatment with CPAP. ${ }^{17}$

Finally, endothelial dysfunction is an important mechanism in the pathophysiology of CVD. ${ }^{18}$ Patients with CVD exhibit impaired endothelium dependent vasodilation reflecting reduced nitric oxide bioavailability. Furthermore, the prognostic significance of impaired endothelium dependent vasodilation has been confirmed in several studies. Individuals with diminished endothelium dependent vasodilation in the coronary or brachial arteries have a higher risk of cardiovascular events. ${ }^{18}$ Drugs such as statins and ACE inhibitors that reduce the risks of CVD also improve endothelial function, suggesting that an improvement in endothelial function may prevent incident CVD. ${ }^{19}$ Endothelium dependent vasodilation (endothelial function) can be measured invasively with brachial or coronary artery catheterisation or non-invasively using a high resolution ultrasound technique of the brachial artery.

In this issue of Thorax Lattimore et al have provided further information concerning the potential benefits of CPAP on cardiovascular physiology. ${ }^{20}$ In this novel study the authors assessed the impact of CPAP treatment on endothelial dysfunction in a small number of patients with moderate to severe OSAH. Endothelial dysfunction was assessed invasively using a brachial artery catheter. Patients had infusions of both acetylcholine (a vasodilator that stimulates release of nitric oxide by the endothelium) and sodium nitroprusside (a vasodilator that directly donates nitric oxide to the endothelium) to assess the degree of endothelium dependent and endothelium independent vasodilation, respectively. After 3 months of CPAP treatment, endothelium dependent vasodilation (acetylcholine mediated) improved from $278 \%$ of baseline pre CPAP to $434 \%$ of baseline post CPAP. Endothelium independent vasodilation (nitroprusside mediated) did not improve significantly. This small study is limited by the lack of a control group, and the results may thus reflect changes secondary to regression to the mean rather than a treatment effect. Nevertheless, these results are consistent with a similar study performed by Ip and associates who studied 28 men with and 28 men without OSAH. ${ }^{21}$ Patients with 
OSAH had impaired flow mediated dilation in response to ischaemia, a marker of endothelial dysfunction, as measured by brachial ultrasonography. Patients with moderate to severe OSAH were randomised to CPAP $(\mathrm{n}=14)$ versus control $(n=13)$ for 4 weeks. The control patients had no significant change in endothelial function while patients on CPAP had a significant increase in brachial artery diameter compared with baseline measurements $(5.1 \% \vee 9.6 \%$ dilation). Nitroglycerin induced dilation, a measure of endothelial independent vasodilation, did not improve significantly ( $15 \% \vee 16.5 \%)$.

At the present time there are no large randomised controlled trials which have examined the impact of long term CPAP treatment in patients with OSAH on more robust CVD outcomes such as incident strokes or heart attacks. However, observational studies have suggested improved CVD outcomes in patients with OSAH using CPAP compared with patients on no treatment Peker and colleagues prospectively followed 182 middle aged men referred for a sleep study with no hypertension or CVD at baseline. ${ }^{22}$ Incident CVD (hypertension, coronary artery disease, stroke, myocardial infarction, arrhythmias) over 7 years occurred in $37 \%$ of patients with OSAH compared with $6.6 \%$ of those without OSAH. CVD events were much more frequent in patients who were incompletely treated for their $\mathrm{OSAH}$ (56\% over 7 years) than in those who were well treated $(6.7 \%)$ Incompletely treated OSAH remained a strong independent risk factor for incident CVD after controlling for potential confounders. Marin and co-workers recently reported on a cohort of patients with varying degrees of sleep disordered breathing (snoring, mild to severe OSAH) and healthy participants who were followed for a mean of 10 years. ${ }^{23}$ Patients with severe untreated OSAH had a much greater risk of developing fatal (odds ratio (OR) $2.87,95 \% \mathrm{CI} 1.17$ to 2.51 ) and non-fatal CVD (OR 3.17, 95\% CI 1.12 to $7.51)$ than healthy controls after adjustment for potential confounding factors. Furthermore, patients with OSAH who were treated with CPAP did not have an increased rate of events (OR 1.05, 95\% CI 0.39 to 2.21 and OR $1.42,95 \%$ CI 0.52 to 3.4 , respectively) compared with healthy controls.

The major limitation of both these studies is their non-randomised design. As with all observational studies, the possibility that the effects seen may be due to an unrecognised confounding factor rather than the effect of treatment cannot be excluded. For instance, it is possible that patients compliant with CPAP may also have been more compliant with lifestyle and pharmacological treatments that could reduce the risks of CVD, and that the benefits seen in patients on CPAP may be a reflection of their compliance with these other activities rather than the effects of CPAP per se.

The study in this issue of Thorax and others provide compelling evidence that CPAP treatment of patients with OSAH improves cardiovascular biochemistry and physiology. This includes improvements in 24 hour blood pressure, lipids, glucose control, systemic inflammation, and endothelial function. These data would certainly support the conclusion that CPAP treatment reduces the risks of developing cardiovascular diseases such as strokes, myocardial infarctions, and sudden cardiac death. However, at present this conclusion is based on circumstantial evidence. The outcomes studied thus far represent surrogate markers, and improvements in them cannot be extrapolated to infer a reduction in CVD events. The effectiveness of drug treatments such as thrombolysis, aspirin, and lipid lowering treatment in reducing CVD have been assessed in large, long term, randomised controlled trials. We now need large, long term, randomised controlled trials of CPAP in patients with OSAH with a composite measure of incident CVD events as the primary outcome. Although the costs of such studies are considerable, they are clearly required given the high prevalence and public health importance of OSAH. Until the results of such studies are available, the basis for treating patients with OSAH with CPAP for a cardiovascular benefit will remain circumstantial.

Thorax 2006;61:459-460.

doi: $10.1136 /$ thx.2005.043836

\section{Authors' affiliations}

N T Ayas, J Fleetham, Sleep Disorders Program and Respiratory Division, University of British Columbia, Vancouver, Canada G B J Mancini, Cardiology Division, University of British Columbia, Vancouver, Canada

Correspondence to: Assistant Professor N T Ayas, Sleep Disorders Program and Respiratory Division, University of British Columbia, Vancouver, Canada; najib.ayas@ vch.ca

\section{REFERENCES}

1 Young $\mathrm{T}$, et al. The occurrence of sleepdisordered breathing among middle-aged adults. $N$ Engl J Med 1993;32:1230-5.

2 Shamsuzzaman AS, Winnicki $M$, Lanfranchi $P$, et al. Elevated $\mathrm{C}$-reactive protein in patients with obstructive sleep apnea. Circulation 2002; 105:2462-4.

3 Kato $M$, Roberts-Thomson P, Phillips BG, et al. Impairment of endothelium-dependent vasodilation of resistance vessels in patients with obstructive sleep apnea. Circulation 2000; 102:2607-10
4 Shahar E, Whitney CW, Redline S, et al. Sleepdisordered breathing and cardiovascular disease: cross-sectional results of the Sleep Heart Health Study. Am J Respir Crit Care Med 2001; 163:19-25.

5 Peppard PE, Young T, Palta M, et al. Prospective study of the association between sleep-disordered breathing and hypertension. N Engl J Med 2000;342:1378-84.

6 Mooe T, Franklin KA, Holmstrom K, et al. Sleepdisordered breathing and coronary artery disease: long-term prognosis. Am J Respir Crit Care Med, 164:1910-3.

7 Malhotra A, Ayas N, Epstein L. The art and science of continuous positive airway pressure therapy in obstructive sleep apnea. Curr Opin Pulm Med 2000;6:490-5.

8 Patel SR, White DP, Malhotra A, et al. The effect of CPAP therapy on subjective and objective sleepiness in obstructive sleep apnea: a metaanalysis of randomized controlled trials. Arch Intern Med 2003; 163:565-71.

9 Pepperell JC, Ramdassingh-Dow S, Crosthwaite $N$, et al. Ambulatory blood pressure after therapeutic and subtherapeutic nasal continuous positive airway pressure for obstructive sleep apnoea: a randomised parallel trial. Lancet 2002:359:204-10.

10 Faccenda JF, Mackay TW, Boon NA, et al. Randomized placebo-controlled trial of continuous positive airway pressure on blood pressure in the sleep apnea-hypopnea syndrome. Am J Respir Crit Care Med 2001; 163:344-8.

11 Becker HF, Jerrentrup A, Ploch T, et al. Effect of nasal continuous positive airway pressure treatment on blood pressure in patients with obstructive sleep apnea. Circulation 2003; 107:68-73.

12 Ridker PM. High-sensitivity C-reactive protein and cardiovascular risk: rationale for screening and primary prevention. Am J Cardiol 2003;92:17-22K.

13 Anon. Randomised trial of cholesterol lowering in 4444 patients with coronary heart disease: the Scandinavian Simvastatin Survival Study (4S). Lancet 1994:344:1383-9.

14 Yokoe T, Minoguchi K, Matsuo H, et al. Elevated levels of C-reactive protein and interleukin- 6 in patients with obstructive sleep apnea syndrome are decreased by nasal continuous positive airway pressure. Circulation 2003; 107: 1129-34.

15 Barcelo A, Barbe F, Llompart E, et al. Effects of obesity on C-reactive protein level and metabolic disturbances in male patients with obstructive sleep apnea. Am J Med 2004;117:1 18-21.

16 Harsch IA, Schahin SP, Radespiel-Troger M, et al. Continuous positive airway pressure treatment rapidly improves insulin sensitivity in patients with obstructive sleep apnea syndrome. Am J Respir Crit Care Med 2004; 169:156-62.

17 Robinson GV, Pepperell JC, Segal HC, et al. Circulating cardiovascular risk factors in obstructive sleep apnoea: data from randomised controlled trials. Thorax 2004:59:777-82.

18 Cohn JN, Quyyumi AA, Hollenberg NK, et al. Surrogate markers for cardiovascular disease: functional markers. Circulation 2004;109(25 Suppl 1):IV31-46.

19 Frick M, Alber HF, Hugel $\mathrm{H}$, et al. Short- and long-term changes of flow-mediated vasodilation in patients under statin therapy. Clin Cardiol 2002;25:291-4

20 Lattimore JL, Wilcox I, Skilton $M$, et al. Treatment of obstructive sleep apnoea leads to improved microvascular endothelial function in the systemic circulation. Thorax 2006;61:491-5.

21 Ip MS, Tse HF, Lam B, et al. Endothelial function in obstructive sleep apnea and response to treatment. Am J Respir Crit Care Med 2004; 169:348-53.

22 Peker Y, Hedner J, et al. Increased incidence of cardiovascular disease in middle-aged men with obstructive sleep apnea: a 7-year follow-up. Am J Respir Crit Care Med 2002;166:159-65.

23 Marin JM, Carrizo SJ, Vincente E, et al. Longterm cardiovascular outcomes in men with obstructive sleep apnoea-hypopnea with or without treatment with continuous positive airway pressure: an observational study. Lancet 2005;365: 1046-53. 